\title{
Extended gravity from noncommutativity
}

\author{
Paolo Aschieri1 \\ Dipartimento di Scienze e Innovazione Tecnologica \\ INFN Gruppo collegato di Alessandria, \\ Università del Piemonte Orientale, \\ Viale T. Michel 11, 15121 Alessandria, Italy \\ aschieri@to.infn.it
}

\begin{abstract}
We review the first order theory of gravity (vierbein formulation) on noncommutative spacetime studied in [1, 2]. The first order formalism allows to couple the theory to fermions. This NC action is then reinterpreted (using the Seiberg-Witten map) as a gravity theory on commutative spacetime that contains terms with higher derivatives and higher powers of the curvature and depend on the noncommutativity parameter $\theta$. When the noncommutativity is switched off we recover the usual gravity action coupled to fermions.

The first nontrival corrections to the usual gravity action coupled to fermions are presented in a manifest Lorentz invariant form.
\end{abstract}

\footnotetext{
${ }^{1}$ The results presented in this Proceedings are based on joint work with Leonardo Castellani.
} 


\section{Introduction}

In the passage from classical mechanics to quantum mechanics classical observables become noncommutative. Similarly we expect that in the passage from classical gravity to quantum gravity, gravity observables, i.e. spacetime itself, with its coordinates and metric structure, will become noncommutative. Thus by formulating Einstein gravity on noncommutative spacetime we may learn some aspects of quantum gravity.

Planck scale noncommutativity is further supported by Gedanken experiments that aim at probing spacetime structure at very small distances. They show that due to gravitational backreaction one cannot test spacetime at those distances. For example, in relativistic quantum mechanics the position of a particle can be detected with a precision at most of the order of its Compton wave length $\lambda_{C}=\hbar / m c$. Probing spacetime at infinitesimal distances implies an extremely heavy particle that in turn curves spacetime itself. When $\lambda_{C}$ is of the order of the Planck length, the spacetime curvature radius due to the particle has the same order of magnitude and the attempt to measure spacetime structure beyond Planck scale fails.

This Gedanken experiment supports finite reductionism. It shows that the description of spacetime as a continuum of points (a smooth manifold) is an assumption no more justified at Planck scale. It is then natural to relax this assumption and conceive a noncommutative spacetime, where uncertainty relations and discretization naturally arise. In this way the dynamical feature of spacetime that prevents from testing sub-Plankian scales is explained by incorporating it at a deeper kinematic level. A similar mechanism happens for example in the passage from Galilean to special relativity. Contraction of distances and time dilatation can be explained in Galilean relativity: they are a consequence of the interaction between ether and the body in motion. In special relativity they become a kinematic feature.

The noncommutative gravity theory we present following [1, 2] is an effective theory that may capture some aspects of a quantum gravity theory. Furthermore we reinterpret space-time noncommutativity as extra interaction terms on commutative spacetime, in this way the theory is equivalent to a higher derivative and curvature extension of Einstein general relativity. We have argued that spacetime noncommutativity should be relevant at Planck scale, however the physical phenomena it induces can also appear at larger scales. For example, due to inflation, noncommutativity of spacetime at inflation scale (that may be as low as Planck scale) can affect cosmological perturbations and possibly the cosmic microwave background spectrum; see for example [3]. We cannot exclude that this noncommutative extension of gravity can be relevant for advancing in our understanding of nowadays open questions in cosmology.

In this contribution, after a short overview of possible noncommutative approaches, we outline the Drinfeld twist approach and review the geometric formulation of theories on noncommutative spacetime [4]. This allows to construct actions invariant under diffeomorphisms. In section 4 we first present usual gravity coupled to fermions in an index free formalism suited for its generalization to the noncommutative case. Then we discuss gauge theories on noncommutative space and in particular local Lorentz symmetry $(S O(3,1)$-gauge symmetry), indeed we need a vierbein formulation of noncommutative gravity in order to couple gravity to spinor fields. The noncommutative Lagrangian coupled to spinor fields is then presented. In section 5 
we reinterpret this NC gravity as an extended gravity theory on commutative spacetime. This is done via the Seiberg-Witten map from noncommutative to commutative gauge fields. The resulting gravity theory then depends on the usual gravitational degrees of freedom plus the noncommutative degrees of freedom, these latter are encoded in a set of mutually commuting vector fields $\left\{X_{I}\right\}$. The leading correction terms to the usual action are explicitly calculated in section 6. They couple spinor fields and their covariant derivatives to derivatives of the curvature tensor and of the vierbein. It is interesting to consider a kinetic term for these vector fields, so that the noncommutative structure of spacetime, as well as its metric structure depend on the matter content of spacetime itself. A model of dynamical noncommutativity is presented in [5]. Noncommutative vierbein gravity can also be coupled to scalar fields [5] and to gauge fields [6].

\section{$2 \quad$ NC geometry approaches}

Before entering the details of the theory, we briefly frame it in the context of noncommutative geometry approaches.

The easiest way to describe a noncommutative spacetime is via the noncommutative algebra of its coordinates, i.e., we give a set of generators and relations. For example

$$
\begin{array}{ll}
{\left[x^{\mu}, x^{\nu}\right]=i \theta^{\mu \nu}} & \text { canonical } \\
{\left[x^{\mu}, x^{\nu}\right]=i f_{\sigma}^{\mu \nu} x^{\sigma}} & \text { Lie algebra } \\
x^{\mu} x^{\nu}-q x^{\nu} x^{\mu}=0 & \text { quantum (hyper)plane }
\end{array}
$$

where $\theta^{\mu \nu}$ (a real antisymmetric matrix), $f_{\sigma}^{\mu \nu}$ (real structure constants) $q$ (a complex number, e.g. a phase) are the respective noncommutativity parameters. Quantum groups and quantum spaces [7, 8] are usually described in this way. In this case we do not have a space (i.e. a set of points), rather we have a noncommutative algebra generated by the coordinates $\left\{x^{\mu}\right\}$ and their relations; when the noncommutativity parameters $\left(\theta^{\mu \nu}, f_{\sigma}^{\mu \nu}, q\right)$ are turned off this algebra becomes commutative and is the algebra of functions on a usual space. Of course we can also impose further constraints, for example periodicity of the coordinates describing the canonical noncommutative spacetime (2.1) (that typical of phase-space quantum mechanics) leads to a noncommutative torus rather than to a noncommutative (hyper)plane. Similarly, constraining the coordinates of the quantum (hyper)plane relations (2.3) we obtain a quantum (hyper)sphere.

This algebraic description should then be complemented by a topological approach. One that for example leads to the notions of continuous functions. This is achieved completing the algebra generated by the noncommutative coordinates to a $C^{\star}$-algebra. Typically $C^{\star}$-algebras arise as algebras of operators on Hilbert space. Connes noncommutative geometry [9] starts from these notions and enriches the $C^{\star}$-algebra structure and its representation on Hilbert space so to generalize to the noncommutative case also the notions of smooth functions and metric structure. 
Another approach is the $\star$-product one. Here we retain the usual space of functions from commutative space to complex numbers, but we deform the pointwise product operation in a $\star$-product one. A $\star$-product sends two functions $(f, g)$ in a third one $(f \star g)$. It is a differential operator on both its arguments (hence it is frequently called a bi-differential operator). It has the associative property $f \star(g \star h)=(f \star g) \star h$. The most known example is the GronewoldMoyal-Weyl star product on $\mathbb{R}^{2 n}$,

$$
(f \star h)(x)=\left.\mathrm{e}^{\frac{i}{2} \theta^{\mu \nu} \frac{\partial}{\partial x^{\mu}} \otimes \frac{\partial}{\partial y^{\nu}}} f(x) h(y)\right|_{x=y} .
$$

Notice that if we set

$$
\mathcal{F}^{-1}=\mathrm{e}^{\frac{i}{2} \theta^{\mu \nu} \frac{\partial}{\partial x^{\mu}} \otimes \frac{\partial}{\partial y^{\nu}}}
$$

then

$$
(f \star h)(x)=\mu \circ \mathcal{F}^{-1}(f \otimes h)(x)
$$

where $\mu$ is the usual product of functions $\mu(f \otimes g)=f h$. The element $\mathcal{F}=\mathrm{e}^{-\frac{i}{2} \theta^{\mu \nu} \frac{\partial}{\partial x^{\mu}} \otimes \frac{\partial}{\partial y^{\nu}}}$ is an example of a Drinfeld twist. It is defined by the exponential series in powers of the noncommutativity parameters $\theta^{\mu \nu}$,

$$
\mathcal{F}=\mathrm{e}^{-\frac{i}{2} \theta^{\mu \nu} \frac{\partial}{\partial x^{\mu}} \otimes \frac{\partial}{\partial y^{\nu}}}=1 \otimes 1-\frac{i}{2} \theta^{\mu \nu} \partial_{\mu} \otimes \partial_{\nu}-\frac{1}{8} \theta^{\mu_{1} \nu_{1}} \theta^{\mu_{2} \nu_{2}} \partial_{\mu_{1}} \partial_{\mu_{2}} \otimes \partial_{\nu_{1}} \partial_{\nu_{2}}+\ldots
$$

It is easy to see that $x^{\mu} \star x^{\nu}-x^{\nu} \star x^{\mu}=i \theta^{m u \nu}$ thus also in this approach we recover the noncommutative algebra (2.1).

In this paper noncommutative spacetime will be spacetime equipped with a $\star$-product. We will not discuss when the exponential series $f \star g=f g-\frac{i}{2} \theta^{\mu \nu} \partial_{\mu}(f) \partial_{\nu}(g)+\ldots$ defining the function $f \star g$ is actually convergent. We will therefore work in the well established context of formal deformation quantization [10]. In the latter part of the paper we will consider a series expansion of the noncommutative gravity action in powers of the noncommutativity parameters $\theta$, we will present the first order in $\theta$ (a second order study appears in [5]), therefore the convergence aspect won't be relevant.

The method of constructing $\star$-products using twists is not the most general method, however it is quite powerful, and the class of $\star$-products obtained is quite wide. For example choosing the appropriate twist we can obtain the noncommutative relations (2.1), (2.2) and also (depending on the structure constant explicit expression) some of the Lie algebra type (2.3).

\section{Twists and $\star-$ Noncommutative Manifolds}

Let $M$ be a smooth manifold, a twist is an invertible element $\mathcal{F} \in U \Xi \otimes U \Xi$ where $U \Xi$ is the universal enveloping algebra of vector fields, (i.e. it is the algebra generated by vector fields on $M$ and where the element $X Y-Y X$ is identified with the vector field $[X, Y])$. The element $\mathcal{F}$ must satisfy some further conditions that we do not write here, but that are satisfied if we consider abelian twists, i.e., twists of the form

$$
\begin{aligned}
\mathcal{F} & =\mathrm{e}^{-\frac{i}{2} \theta^{I J} X_{I} \otimes X_{J}} \\
& =1 \otimes 1-\frac{i}{2} \theta^{I J} X_{I} \otimes X_{J}-\frac{1}{8} \theta^{I_{1} J_{1}} \theta^{I_{2} J_{2}} X_{I_{1}} X_{I_{2}} \otimes X_{J_{1}} X_{J_{2}}+\ldots
\end{aligned}
$$


were the vector fields $X_{I}(I=1, \ldots s$ with $s$ not necessarily equal to $m=\operatorname{dim} \mathrm{M})$ are mutually commuting $\left[X_{I}, X_{J}\right]=0$ (hence the name abelian twist).

It is convenient to introduce the following notation

$$
\begin{aligned}
\mathcal{F}^{-1} & =1 \otimes 1+\frac{i}{2} \theta^{I J} X_{I} \otimes X_{J}-\frac{1}{8} \theta^{I_{1} J_{1}} \theta^{I_{2} J_{2}} X_{I_{1}} X_{I_{2}} \otimes X_{J_{1}} X_{J_{2}}+\ldots \\
& =\overline{\mathrm{f}}^{\alpha} \otimes \overline{\mathrm{f}}_{\alpha}
\end{aligned}
$$

where a sum over the multi-index $\alpha$ is understood.

Let $\mathcal{A}$ be the algebra of smooth functions on the manifold $M$. Then, given a twist $\mathcal{F}$, we deform $\mathcal{A}$ in a noncommutative algebra $\mathcal{A}_{\star}$ by defining the new product of functions

$$
f \star h=\overline{\mathrm{f}}^{\alpha}(f) \overline{\mathrm{f}}_{\alpha}(h),
$$

we see that this formula is a generalization of the Gronewold-Moyal-Weyl star product on $\mathbb{R}^{2 n}$ defined in (2.4). Since the vector fields $X_{I}$ are mutually commuting then this $\star$-product is associative. Note that only the algebra structure of $\mathcal{A}$ is changed to $\mathcal{A}_{\star}$ while, as vector spaces, $\mathcal{A}$ and $\mathcal{A}_{\star}$ are the same. We similarly consider the algebra of exterior forms $\Omega^{\bullet}$ with the wedge product $\wedge$, and deform it in the noncommutative exterior algebra $\Omega_{\star}^{\bullet}$ that is characterized by the graded noncommutative exterior product $\wedge_{\star}$ given by

$$
\tau \wedge_{\star} \tau^{\prime}=\overline{\mathrm{f}}^{\alpha}(\tau) \wedge \overline{\mathrm{f}}_{\alpha}\left(\tau^{\prime}\right)
$$

where $\tau$ and $\tau^{\prime}$ are arbitrary exterior forms. Notice that the action of the twist on $\tau$ and $\tau^{\prime}$ is via the Lie derivative: each vector field $X_{I_{1}}, X_{I_{2}}, X_{J_{1}}, X_{J_{2}} \ldots$ in (3.5) acts on forms via the Lie derivative.

It is not difficult to show that the usual exterior derivative is compatible with the new $\wedge_{\star}$-product,

$$
d\left(\tau \wedge_{\star} \tau^{\prime}\right)=d(\tau) \wedge_{\star} \tau^{\prime}+(-1)^{\operatorname{deg}(\tau)} \tau \wedge_{\star} d \tau^{\prime}
$$

this is so because the Lie derivative commutes with the exterior derivative.

We also have compatibility with the usual undeformed integral (graded cyclicity property):

$$
\int \tau \wedge_{\star} \tau^{\prime}=(-1)^{\operatorname{deg}(\tau) \operatorname{deg}\left(\tau^{\prime}\right)} \int \tau^{\prime} \wedge_{\star} \tau
$$

(the equality holds up to boundary terms). Finally we also have compatibility with the undeformed complex conjugation:

$$
\left(\tau \wedge_{\star} \tau^{\prime}\right)^{*}=(-1)^{\operatorname{deg}(\tau) \operatorname{deg}\left(\tau^{\prime}\right)} \tau^{*} \wedge_{\star} \tau^{*}
$$

Note. We remark that all these properties are due to the special nature of the $\star$-product we consider. As shown in [10] $\star$-products are in 1-1 correspondence with Poisson structures $\{$, on the manifold $M$. The Poisson structure the twist $\mathcal{F}$ induces is $\{f, g\}=i \theta^{I J} X_{I}(f) X_{J}(g)$. However the twist $\mathcal{F}$ encodes more information than the Poisson bracket $\{$,$\} . The key point$ is that the twist is associated with the Lie algebra $\Xi$ (and morally with the diffeomorphisms group of $M$ ). Given a twist we can deform the Lie algebra $\Xi$ (the diffeomorphisms group of $M$ ) and then, using the Lie derivative action (the action of the diffeomorphisms group) we induce noncommutative deformations of the algebra of functions on $M$, of the exterior algebra and more generally of the differential and Riemannian geometry structures on $M$ [11], leading to noncommutative Einstein equations for the metric tensor [11]. 


\section{Noncommutative vierbein gravity coupled to fermions}

\subsection{Classical action}

The usual action of first-order gravity coupled to spin $\frac{1}{2}$ fields reads:

$$
S=\varepsilon_{a b c d} \int R^{a b} \wedge V^{c} \wedge V^{d}-i \bar{\psi} \gamma^{a} V^{b} \wedge V^{c} \wedge V^{d} \wedge D \psi-i(D \bar{\psi}) \gamma^{a} \wedge V^{b} \wedge V^{c} \wedge V^{d} \psi
$$

with $R^{a b}=d \omega^{a b}-\omega^{a}{ }_{c} \wedge \omega^{c b}$, and the Dirac conjugate defined as usual: $\bar{\psi}=\psi^{\dagger} \gamma_{0}$. This action can be recast in an index-free form [12], 11, convenient for generalization to the noncommutative case:

$$
S=\int \operatorname{Tr}\left(i R \wedge V \wedge V \gamma_{5}\right)+\bar{\psi} V \wedge V \wedge V \gamma_{5} D \psi+D \bar{\psi} \wedge V \wedge V \wedge V \gamma_{5} \psi
$$

where

$$
R=d \Omega-\Omega \wedge \Omega, \quad D \psi=d \psi-\Omega \psi, \quad D \bar{\psi}=\overline{D \psi}=d \bar{\psi}+\bar{\psi} \Omega
$$

with

$$
\Omega \equiv \frac{1}{4} \omega^{a b} \gamma_{a b}, \quad V \equiv V^{a} \gamma_{a}, \quad R \equiv \frac{1}{4} R^{a b} \gamma_{a b}
$$

taking value in Dirac gamma matrices. Use of the gamma matrix identities $\gamma_{a b c}=i \varepsilon_{a b c d} \gamma^{d} \gamma_{5}$, $\operatorname{Tr}\left(\gamma_{a b} \gamma_{c} \gamma_{d} \gamma_{5}\right)=-4 i \varepsilon_{a b c d}$ in computing the trace leads back to the usual action (4.1).

The action (4.2) is invariant under local diffeomorphisms (because it is the integral of a 4 -form on a 4-manifold) and under local Lorentz rotations. In the index-free form they read

$$
\delta_{\varepsilon} V=-[V, \varepsilon], \quad \delta_{\varepsilon} \Omega=d \varepsilon-[\Omega, \varepsilon], \quad \delta_{\varepsilon} \psi=\varepsilon \psi, \quad \delta_{\varepsilon} \bar{\psi}=-\bar{\psi} \varepsilon
$$

with $\varepsilon=\frac{1}{4} \varepsilon^{a b} \gamma_{a b}$ The local Lorentz invariance of the index free action follows from $\delta_{\varepsilon} R=-[R, \varepsilon]$ and $\delta_{\varepsilon} D \psi=\varepsilon D \psi$, the cyclicity of the trace $\operatorname{Tr}$ and the fact that the gauge parameter $\varepsilon$ commutes with $\gamma_{5}$.

\subsection{Noncommutative gauge theory and Lorentz group}

Consider an infinitesimal gauge transformation $\lambda=\lambda^{A} T^{A}$, where the generators $T^{A}$ belong to some representation (the fundamental, the adjoint, etc.) of a Lie group $G$. Since two consecutive gauge transformations are a gauge transformation, the commutator of two infinitesimal ones $\left[\lambda, \lambda^{\prime}\right]$, closes in the Lie algebra of $G$. This in general is no more the case in noncommutative gauge theories. In the noncommutative case the commutator of two infinitesimal gauge transformations is

$$
\left[\lambda \star \lambda^{\prime}\right] \equiv \lambda \star \lambda^{\prime}-\lambda^{\prime} \star \lambda=\frac{1}{2}\left\{\lambda^{A} \star \lambda^{\prime B}\right\}\left[T^{A}, T^{B}\right]+\frac{1}{2}\left[\lambda^{A} \star \lambda^{\prime B}\right]\left\{T^{A}, T^{B}\right\} .
$$

We see that also the anticommutator $\left\{T^{A}, T^{B}\right\}$ appears. This is fine if our gauge group is for example $U(N)$ or $G L(N)$ in the fundamental or in the adjoint, since in this case $\left\{T^{A}, T^{B}\right\}$ is 
again in the Lie algebra, however for more general Lie algebras (including all simple Lie algebras) we have to enlarge the Lie algebra to include also anticommutators besides commutators, i.e. we have to consider all possible products $T^{A} T^{B} \ldots T^{C}$ of generators.

Our specific case is the Lorentz group in the spinor representation given by the Dirac gamma matrices $\gamma_{a b}$. The algebra generated by these gamma matrices is that of all even $4 \times 4$ gamma matrices. The noncommutative gauge parameter will therefore have components

$$
\varepsilon=\frac{1}{4} \varepsilon^{a b} \gamma_{a b}+i \varepsilon \mathbb{1}+\tilde{\varepsilon} \gamma_{5}
$$

The extra gauge parameters $\varepsilon, \tilde{\varepsilon}$ can be chosen to be real (like $\varepsilon_{a b}$ ). Indeed the reality of $\varepsilon_{a b}$, $\varepsilon, \tilde{\varepsilon}$ is equivalent to the hermiticity condition

$$
-\gamma_{0} \varepsilon \gamma_{0}=\varepsilon^{\dagger}
$$

and if the gauge parameters $\varepsilon, \varepsilon^{\prime}$ satisfy this condition then also $\left[\varepsilon \star \varepsilon^{\prime}\right]$ is easily seen to satisfy this hermiticity condition.

We have centrally extended the Lorentz group to

$$
S O(3,1) \rightarrow S O(3,1) \times U(1) \times R^{+}
$$

or more precisely, (since our manifold $M$ has a spin structure and we have a gauge theory of the spin group $S L(2, C)$ )

$$
S L(2, C) \rightarrow G L(2, C)
$$

The Lie algebra generator $i \mathbb{1}$ is the anti-hermitian generator corresponding to the $U(1)$ extension, while $\gamma_{5}$ is the hermitian generator corresponding to the noncompact $R^{+}$extension.

Since under noncommutative gauge transformations we have

$$
\delta_{\varepsilon} \Omega=d \varepsilon-\Omega \star \varepsilon+\varepsilon \star \Omega
$$

also the spin connection and the curvature will be valued in the $G L(2, C)$ Lie algebra representation given by all the even gamma matrices,

$$
\Omega=\frac{1}{4} \omega^{a b} \gamma_{a b}+i \omega \mathbb{1}+\tilde{\omega} \gamma_{5}, \quad R=\frac{1}{4} R^{a b} \gamma_{a b}+i r \mathbb{1}+\tilde{r} \gamma_{5}
$$

Similarly the gauge transformation of the vierbein,

$$
\delta_{\varepsilon} V=-V \star \varepsilon+\varepsilon \star V
$$

closes in the vector space of odd gamma matrices (i.e. the vector space linearly generated by $\left.\gamma^{a}, \gamma^{a} \gamma_{5}\right)$ and not in the subspace of just the $\gamma^{a}$ matrices. Hence the noncommutative vierbein are valued in the odd gamma matrices

$$
V=V^{a} \gamma_{a}+\tilde{V}^{a} \gamma_{a} \gamma_{5}
$$




\subsection{Noncommutative Gravity action and its symmetries}

We have all the ingredients in order to generalize to the noncommutative case the gravity action coupled to spinors of Section 3: an abelian twist giving the star products of functions and forms on the spacetime manifold $M$ (and compatible with usual integration on $M$ ); an extension to $G L(2, C)$ of the Lorentz gauge group, so that infinitesimal noncommutative gauge transformations close in this extended Lie algebra. The action reads

$$
S=\int \operatorname{Tr}\left(i R \wedge_{\star} V \wedge_{\star} V \gamma_{5}\right)+\bar{\psi} \star V \wedge_{\star} V \wedge_{\star} V \wedge_{\star} \gamma_{5} D \psi+D \bar{\psi} \wedge_{\star} V \wedge_{\star} V \wedge_{\star} V \star \gamma_{5} \psi
$$

with

$$
R=d \Omega-\Omega \wedge_{\star} \Omega, \quad D \psi=d \psi-\Omega \star \psi, \quad D \bar{\psi}=d \bar{\psi}+\bar{\psi} \star \Omega .
$$

\section{Gauge invariance}

The invariance of the noncommutative action (4.10) under the $\star$-variations is demonstrated in exactly the same way as for the commutative case: noting that besides (4.6) and (4.8) we have

$$
\delta_{\varepsilon} \psi=\varepsilon \star \psi, \quad \delta_{\varepsilon} \bar{\psi}=-\bar{\psi} \star \varepsilon, \quad \delta_{\varepsilon} D \psi=\varepsilon \star D \psi, \quad \delta_{\varepsilon} D \bar{\psi}=-D \bar{\psi} \star \varepsilon, \quad \delta_{\varepsilon} R=-R \star \varepsilon+\varepsilon \star R,
$$

and using that $\varepsilon$ commutes with $\gamma_{5}$, and the cyclicity of the trace together with the graded cyclicity of the integral with respect to the $\star$-product.

\section{Diffeomorphisms invariance}

The $\star$-action (4.10) is invariant under usual diffeomorphisms, being the integral of a 4-form. Under these diffeomorphisms the vector fields $X_{I}$ transform covariantly. We also mention that since the vector fields $X_{I}$ appear only in the $\star$-product, the action is furthermore invariant under $\star$-diffeomorphisms as defined in [11], see discussion in [4], Section 8.2.4. Under these deformed diffeomorphisms the vector fields $X_{I}$ do not transform.

\section{Reality of the action}

Hermiticity conditions can be imposed on the fields $V$ and $\Omega$ as done with the gauge parameter $\varepsilon$ in (4.5) :

$$
\gamma_{0} V \gamma_{0}=V^{\dagger}, \quad-\gamma_{0} \Omega \gamma_{0}=\Omega^{\dagger}
$$

These hermiticity conditions are consistent with the gauge variations and can be used to check that the action (4.10) is real by comparing it to its complex conjugate (obtained by taking the Hermitian conjugate of the 4-form inside the trace in the integral). As previously observed for the component gauge parameters $\varepsilon^{a b}, \varepsilon, \tilde{\varepsilon}$, the hermiticity conditions (4.13) imply that the component fields $V^{a}, \tilde{V}^{a}, \omega^{a b}, \omega$, and $\tilde{\omega}$ are real fields.

\section{Charge conjugation invariance}

Noncommutative charge conjugation is the following transformation (extended linearly and multiplicatively $\left.{ }^{2}\right)$ :

$\psi \rightarrow \psi^{C} \equiv C(\bar{\psi})^{T}=-\gamma_{0} C \psi^{*}, \quad V \rightarrow V^{C} \equiv C V^{T} C, \quad \Omega \rightarrow \Omega^{C} \equiv C \Omega^{T} C, \star_{\theta} \rightarrow \star_{\theta}^{C}=\star_{-\theta}$,

\footnotetext{
${ }^{2}$ Multiplicativity and the transformation $\star \rightarrow \star_{\theta}^{C}=\star_{-} \theta$ is equivalent to antimultiplicativity with respect to the $\star$-product: $(f \star g)^{C}=g^{C} \star f^{C}$ (for $f$ and $g$ scalar fields that are not matrix valued).
} 
and consequently $\wedge_{\star_{\theta}} \rightarrow \wedge_{\star_{\theta}}^{C}=\wedge_{\star_{-} \theta}$. Then the action (4.10) is invariant under charge conjugation. For example

$$
\begin{aligned}
S_{\text {bosonic }}^{C} & =i \int \operatorname{Tr}\left(R^{C} \wedge_{-\theta} V^{C} \wedge_{-\theta} V^{C} \gamma_{5}\right)^{T}=-i \int \operatorname{Tr}\left(R^{T} \wedge_{-\theta} V^{T} \wedge_{-\theta} V^{T} C \gamma_{5} C^{-1}\right)^{T} \\
& =-i \int \operatorname{Tr}\left(\left(V^{T} \wedge_{-\theta} V^{T} \gamma_{5}^{T}\right)^{T} \wedge_{\star} R\right)=-i \int \operatorname{Tr}\left(-\left(V^{T} \gamma_{5}^{T}\right)^{T} \wedge_{\star} V \wedge_{\star} R\right) \\
& =i \int \operatorname{Tr}\left(\gamma_{5} V \wedge_{\star} V \wedge_{\star} R\right)=i \int \operatorname{Tr}\left(R \wedge_{\star} \gamma_{5} V \wedge_{\star} V\right)=i \int \operatorname{Tr}\left(R \wedge_{\star} V \wedge_{\star} V \gamma_{5}\right) \\
& =S_{\text {bosonic }}
\end{aligned}
$$

Similarly the fermionic part of the action satisfies $S_{\text {fermionic }}^{C}=S_{\text {fermionic }}$.

\section{Charge conjugation conditions}

In the classical limit $\theta \rightarrow 0$ the $\star$-product becomes the usual pointwise product. The noncommutative gauge symmetry becomes a usual gauge symmetry with gauge group $G L(2, C)$ and the noncommutative vierbein in the classical limit leads to two independent vierbeins: $V^{a}$ and $\tilde{V}^{a}$ transforming both only under the $S L(2, C)$ subgroup of $G L(2, C)$. As observed in [12] this is problematic because we obtain two massless gravitons, and only one local Lorentz symmetry, that is not enough in order to kill the unphysical degrees of freedom. Either we concoct a mechanism such that the second graviton becomes massive or we further constraint the noncommutative theory so that in the classical limit the extra vierbein vanishes.

The vanishing of the $\tilde{V}^{a}$ components in the classical limit is achieved by imposing charge conjugation conditions on the fields [1]:

$$
C V_{\theta}(x) C=V_{-\theta}(x)^{T}, \quad C \Omega_{\theta}(x) C=\Omega_{-\theta}(x)^{T}, \quad C \varepsilon_{\theta}(x) C=\varepsilon_{-\theta}(x)^{T}
$$

These conditions involve the $\theta$-dependence of the fields. This latter is due to the $\star$-product $\theta$-dependence (recall that the $\star$-product is defined as an expansion in power series of the noncommutativity parameter $\theta$ ). Since noncommutative gauge transformations involve the $\star$-product, the gauge transformed fields will be $\theta$-dependent and hence field configurations are in general $\theta$-dependent.

Conditions (4.16) are consistent with the $\star$-gauge transformations. For example the field $C V_{\theta}(x)^{T} C$ can be shown to transform in the same way as $V_{-\theta}(x)$ [1].

For the component fields and gauge parameters the charge conjugation conditions imply that the components $V^{a}, \omega^{a b}$ are even in $\theta$, while the components $\tilde{V}^{a}, \omega, \tilde{\omega}$ are odd:

$$
\begin{aligned}
V_{\theta}^{a} & =V_{-\theta}^{a}, \quad \omega_{\theta}^{a b}=\omega_{-\theta}^{a b} \\
\tilde{V}_{\theta}^{a} & =-\tilde{V}_{-\theta}^{a}, \quad \omega_{\theta}=-\omega_{-\theta}, \quad \tilde{\omega}_{\theta}=-\tilde{\omega}_{-\theta} .
\end{aligned}
$$

Similarly for the gauge parameters: $\varepsilon_{\theta}^{a b}=\varepsilon_{-\theta}^{a b}, \quad \varepsilon_{\theta}=-\varepsilon_{-\theta}, \quad \tilde{\varepsilon}_{\theta}=-\tilde{\varepsilon}_{-\theta}$. In particular, since the components $\tilde{V}^{a}$ are odd in $\theta$ we achieve their vanishing in the classical limit.

We can also conclude that the bosonic action is even in $\theta$. Indeed (4.16) implies

$$
V^{C}=V_{-\theta}, \quad \Omega^{C}=\Omega_{-\theta}, \quad R^{C}=R_{-\theta} .
$$


Hence the bosonic action $S_{\text {bosonic }}(\theta)$ is mapped into $S_{\text {bosonic }}(-\theta)$ under charge conjugation. Also for the fermionic action, $S_{\text {fermionic }}(\theta)$, we have $S_{\text {fermionic }}(\theta)^{C}=S_{\text {fermionic }}(-\theta)$ if the fermions are Majorana, i.e. if they satisfy $\psi^{C}=\psi_{-\theta}$. From $S_{\text {bosonic }}(\theta)=S_{\text {bosonic }}(-\theta)$ we conclude that all noncommutative corrections to the classical action of pure gravity are even in $\theta$; this is also the case if we couple noncommutative gravity to Majorana fermions.

\section{$5 \quad$ Seiberg-Witten map (SW map)}

In the previous section we have formulated a noncommutative gravity theory that in the classical limit $\theta \rightarrow 0$ reduces to usual vierbein gravity. In the full noncommutative regime it has however a doubling of the vierbein fields. We can insist on a noncommutative gravity theory that has the same degrees of freedom of the classical one. This is doable if we use the Seiberg-Witten map to express the noncommutative fields in terms of the commutative ones. In this way the gauge group is the Lorentz group $S L(2, C)$ and not the centrally extended one $G L(2, C)$, indeed also the noncommutative gauge parameters $\varepsilon^{a b}, \varepsilon, \tilde{\varepsilon}$ are expressed in term of the commutative ones $\varepsilon^{a b}$.

Because of the SW map the noncommutative fields can therefore be expanded in terms of the commutative ones, and hence the noncommutative gravity action can be expanded, order by order in powers of $\theta$, in terms of the usual commutative gravity and spinor field as well as of the noncommutativity vector fields $X_{I}$. As we will see, we thus obtain a commutative gravity action that at zeroth order in $\theta$ is usual gravity (coupled to spinors) and at higher orders in $\theta$ contains higher derivative terms describing gravity and spinor fields coupled to the noncommutativity vector fields $X_{I}$.

The Seiberg-Witten map (SW map) relates the noncommutative gauge fields $\Omega$ to the ordinary $\Omega^{0}$, and the noncommutative gauge parameters $\varepsilon$ to the ordinary $\varepsilon^{0}$ and $\Omega^{0}$ so as to satisfy:

$$
\Omega\left(\Omega^{0}\right)+\delta_{\varepsilon} \Omega\left(\Omega^{0}\right)=\Omega\left(\Omega^{0}+\delta_{\varepsilon^{0}} \Omega^{0}\right)
$$

with the noncommutative and ordinary gauge variations given by

$$
\delta_{\varepsilon} \Omega=\mathrm{d} \varepsilon-\Omega \star \varepsilon+\varepsilon \star \Omega, \quad \delta_{\varepsilon^{0}} \Omega^{0}=\mathrm{d} \varepsilon^{0}-\Omega^{0} \varepsilon^{0}+\varepsilon^{0} \Omega^{0} .
$$

Equation (5.20) can be solved order by order in $\theta$ [13], yielding $\Omega$ and $\varepsilon$ as power series in $\theta$ :

$$
\begin{aligned}
\Omega\left(\Omega^{0}, \theta\right) & =\Omega^{0}+\Omega^{1}\left(\Omega^{0}\right)+\Omega^{2}\left(\Omega^{0}\right)+\cdots+\Omega^{n}\left(\Omega^{0}\right)+\cdots \\
\varepsilon\left(\varepsilon^{0}, \Omega^{0}, \theta\right) & =\varepsilon^{0}+\varepsilon^{1}\left(\varepsilon^{0}, A^{0}\right)+\varepsilon^{2}\left(\varepsilon^{0}, \Omega^{0}\right)+\cdots+\varepsilon^{n}\left(\varepsilon^{0}, \Omega^{0}\right)+\cdots
\end{aligned}
$$

where $\Omega^{n}\left(\Omega^{0}\right)$ and $\varepsilon^{n}\left(\varepsilon^{0}, \Omega^{0}\right)$ are of order $n$ in $\theta$. Note that $\varepsilon$ depends on the ordinary $\varepsilon^{0}$ and also on $\Omega^{0}$.

The Seiberg-Witten condition (5.20) states that the dependence of the noncommutative gauge field on the ordinary one is fixed by requiring that ordinary gauge variations of $\Omega^{0}$ inside $\Omega\left(\Omega^{0}\right)$ produce the noncommutative gauge variation of $\Omega$. This implies that once we expand, order by order in $\theta$, the noncommutative action in terms of the commutative fields, the resulting action will be gauge invariant under ordinary local Lorentz transformations because the 
noncommutative action is invariant under the local noncommutative Lorentz transformations of Section 4.3 .

Similarly for matter fields we have $\psi\left(\psi^{0}, \Omega^{0}\right)+\delta_{\varepsilon} \psi\left(\psi^{0}, \Omega^{0}\right)=\psi\left(\psi^{0}+\delta_{\varepsilon^{0}} \psi^{0}, \Omega^{0}+\delta_{\varepsilon^{0}} \Omega^{0}\right)$ so that again noncommutative gauge variations correspond to commutative ones.

Following ref. [2, up to first order in $\theta$ the solution to the Seiberg-Witten conditions reads:

$$
\begin{aligned}
& \Omega=\Omega^{0}+\frac{i}{4} \theta^{I J}\left\{\Omega_{I}^{0}, \ell_{J} \Omega^{0}+R_{J}^{0}\right\} \\
& \varepsilon=\varepsilon^{0}+\frac{i}{4} \theta^{I J}\left\{\Omega_{I}^{0}, \ell_{J} \varepsilon^{0}\right\} \\
& R=R^{0}+\frac{i}{4} \theta^{I J}\left(\left\{\Omega_{I}^{0},\left(\ell_{J}+L_{J}\right) R^{0}\right\}-\left[R_{I}^{0}, R_{J}^{0}\right]\right) \\
& \psi=\psi^{0}+\frac{i}{4} \theta^{I J} \Omega_{I}^{0}\left(\ell_{J}+L_{J}\right) \psi^{0}
\end{aligned}
$$

where $\Omega_{A}^{0}, R_{A}^{0}$ are defined as the contraction along the tangent vector $X_{I}$ of the exterior forms $\Omega^{0}, R^{0}$, i.e. $\Omega_{A}^{0} \equiv i_{I} \Omega^{0}, R_{I}^{0} \equiv i_{I} R^{0}$, $\left(i_{I}\right.$ being the contraction along $\left.X_{I}\right)$. We have also introduced the Lie derivative $\ell_{I}$ along the vector field $X_{I}$, and the covariant Lie derivative $L_{I}$ along the vector field $X_{I} . L_{I}$ acts on $R^{0}$ and $\psi^{0}$ as $L_{I} R^{0}=\ell_{I} R^{0}-\Omega_{B}^{0} \star R^{0}+R^{0} \star \Omega_{I}^{0}$ and $L_{I} \psi^{0}=\ell_{I} \psi^{0}-\Omega_{I}^{0} \psi^{0}$. In fact the covariant Lie derivative $L_{I}$ has the Cartan form:

$$
L_{I}=i_{I} D+D i_{I}
$$

where $D$ is the covariant derivative. We refer to [2] for higher order in $\theta$ expressions. All these formulae are not $S O(1,3)$-gauge covariant, due to the presence of the "naked" connection $\Omega^{0}$ and the non-covariant Lie derivative $\ell_{I}=i_{I} \mathrm{~d}+\mathrm{d} i_{I}$. However, when inserted in the NC action the resulting action is gauge invariant order by order in $\theta$. Indeed usual gauge variations induce the $\star$-gauge variations under which the $\mathrm{NC}$ action is invariant. Therefore the $\mathrm{NC}$ action, re-expressed in terms of ordinary fields via the SW map, is invariant under usual gauge transformations. Since these do not involve $\theta$, the expanded action is invariant under ordinary gauge variations order by order in $\theta$. Moreover the action, once re-expressed in terms of ordinary fields remains geometric, and hence invariant under diffeomorphisms. This is the case because the noncommutative action and the SW map are geometric, we indeed see that only coordinate independent operations like the contraction $i_{I}$ and the Lie derivatives $\ell_{I}$ and $L_{I}$ appear in the SW map.

From (5.24) and (5.27) we also deduce

$$
D \psi=D \psi^{0}+\frac{i}{4} \theta^{I J}\left(\Omega_{I}^{0}\left(\ell_{J}+L_{J}\right) D \psi^{0}-2 R_{I} L_{J} \psi\right) .
$$

\section{Action at first order in $\theta$}

The expression of the gravity action, up to second order in $\theta$, in terms of the commutative fields and of the first order fields (5.24) -(5.28) has been given in [2]. The action is gauge invariant even if the expression in [2] is not explicitly gauge invariant. We here present the explicit gauge invariant expression for the action up to first order in $\theta$. We replace the noncommutative fields 
appearing in the action with their expansions (5.24)-(5.28) in commutative fields and integrate by parts in order to obtain an explicit $S O(3,1)$ gauge invariant action. We thus obtain the following gravity action coupled to spinors

$$
\begin{aligned}
S= & \operatorname{Tr}\left(i R V V \gamma_{5}\right)+\bar{\psi} V^{3} \gamma_{5} D \psi+D \bar{\psi} V^{3} \gamma_{5} \psi \\
& +\frac{i}{4} \theta^{I J}\left(\bar{\psi}\left\{V^{3}, R_{I J}\right\} \gamma_{5} D \psi+D \bar{\psi}\left\{V^{3}, R_{I J}\right\} \gamma_{5} \psi\right) \\
& +\frac{i}{2} \theta^{I J}\left(2 L_{I} \bar{\psi} R_{J} V^{3} \gamma_{5} \psi-2 \bar{\psi} V^{3} R_{I} \gamma_{5} L_{J} \psi-L_{I} \bar{\psi} V^{3} \gamma_{5} L_{J} D \psi-L_{I} D \bar{\psi} V^{3} \gamma_{5} L_{J} \psi\right. \\
& \left.+\bar{\psi}\left(\left\{L_{I} V L_{J} V, V\right\}+L_{I} V V L_{J} V\right) \gamma_{5} D \psi+D \bar{\psi}\left(\left\{L_{I} V L_{J} V, V\right\}+L_{I} V V L_{J} V\right) \gamma_{5} \psi\right)+O\left(\theta^{2}\right)
\end{aligned}
$$

where with obvious abuse of notation we have omitted the apex ${ }^{0}$ denoting commutative fields, we also have omitted writing the wedge product, and $V^{3}=V \wedge V \wedge V$.

\section{Conclusions}

We have constructed an extended Einstein gravity action that: i) is explicitly invariant under local Lorentz transformations because expressed solely in terms of the gauge covariant operators $L_{I}, i_{I}, D$ and fields $R, V, \psi$; ii) is diffeomorphic invariant; iii) has the same fields of classical gravity plus the noncommutative structure. This latter is given by the vector fields $\left\{X_{I}\right\}$ that choosing an appropriate kinetic term can become dynamical, the idea being that both spacetime curvature and noncommutativity should depend on matter distribution.

This extended action has been obtained from considering gravity on noncommutative spacetime, that as argued in the introduction is a very natural assumption at high energies, like those close to the inflationary epoch.

\section{Acknowledgements}

We acknowledge the fruitful and pleasant atmosphere and the perfect organization enjoyed during the Udine Symposium.

\section{A Gamma matrices in $D=4$}

We summarize in this Appendix our gamma matrix conventions in $D=4$.

$$
\begin{aligned}
& \eta_{a b}=(1,-1,-1,-1), \quad\left\{\gamma_{a}, \gamma_{b}\right\}=2 \eta_{a b}, \quad\left[\gamma_{a}, \gamma_{b}\right]=2 \gamma_{a b}, \\
& \gamma_{5} \equiv i \gamma_{0} \gamma_{1} \gamma_{2} \gamma_{3}, \quad \gamma_{5} \gamma_{5}=1, \quad \varepsilon_{0123}=-\varepsilon^{0123}=1 \\
& \gamma_{a}^{\dagger}=\gamma_{0} \gamma_{a} \gamma_{0}, \quad \gamma_{5}^{\dagger}=\gamma_{5} \\
& \gamma_{a}^{T}=-C \gamma_{a} C^{-1}, \quad \gamma_{5}^{T}=C \gamma_{5} C^{-1}, \quad C^{2}=-1, \quad C^{\dagger}=C^{T}=-C
\end{aligned}
$$




\section{References}

[1] P. Aschieri and L. Castellani, "Noncommutative D=4 gravity coupled to fermions," JHEP 0906 (2009) 086 [arXiv:0902.3817 [hep-th]].

[2] P. Aschieri and L. Castellani, "Noncommutative gravity coupled to fermions: second order expansion via Seiberg-Witten map," arXiv:1111.4822 [hep-th].

[3] F. Lizzi, G. Mangano, G. Miele and M. Peloso, "Cosmological perturbations and short distance physics from noncommutative geometry," JHEP 0206 (2002) 049 hep-th/0203099.

[4] P. Aschieri, M. Dimitrijevic, P. Kulish, F. Lizzi, J. Wess, "Noncommutative Spacetimes", Lecture Notes in Physics, vol. 774, Springer 2009.

[5] P. Aschieri and L. Castellani, "Extended gravity theories from dynamical noncommutativity," arXiv:1206.4096 [hep-th].

[6] P. Aschieri and L. Castellani, "Noncommutative gauge fields coupled to noncommutative gravity," arXiv:1205.1911 [hep-th].

[7] L.D. Faddeev, N.Yu. Reshetikhin, L.A. Takhtajan, Quantization of Lie groups and Lie algebras, Algebra i Anal. 11 (1989) 178 (Leningrad Math. J. 1193 (1990)).

[8] Yu.Manin, Quantum groups and non-commutative geometry, Preprint Montreal Univ., CRM-1561 (1988);

[9] A. Connes, "Non-commutative geometry." Academic Press, San Diego, CA, 1994, 661 p., ISBN 0-12-185860-X.

[10] M. Kontsevich, "Deformation quantization of Poisson manifolds. 1.," Lett. Math. Phys. 66 (2003) 157 arXiv:q-alg/9709040 [q-alg]].

[11] P. Aschieri, M. Dimitrijević, F. Meyer and J. Wess, Noncommutative Geometry and Gravity, Class. Quant. Grav. 23, 1883-1912 (2006), hep-th/0510059.

[12] A. H. Chamseddine, "Sl(2,C) gravity with complex vierbein and its noncommutative extension," Phys. Rev. D 69, 024015 (2004) arXiv:hep-th/0309166.

[13] K. Ulker, B. Yapiskan, "Seiberg-Witten maps to all orders," Phys. Rev. D77 (2008) 065006. [arXiv:0712.0506 [hep-th]]. 